\title{
SPH MODEL TO SIMULATE MOVEMENT OF GRASS MEADOW OF POSIDONIA UNDER WAVES
}

\author{
Thibault Oudart ${ }^{1}$ and Philippe Larroudé ${ }^{2}$
}

\begin{abstract}
The objective of this paper is to try a new approach to simulate the interactions between waves and algae. The chosen method is to simulate waves and plants through SPH (Smoothed Particle Hydrodynamics, SPH). In this model, the algae are defined as a solid that respects Hook's law, which is in direct interaction with the fluid part. Given the properties of this method especially in terms of computation time, the dimensions of the simulations are limited. A successful representation of the movement of algae under waves or/and current by SPH will permit the determination of coefficient of friction corresponding to a type of algae, that can be used in a different larger scale code.
\end{abstract}

Keywords: Fluid-Structure interaction, SPH model, Wave attenuation, Numerical movement of aquatic plant

\section{INTRODUCTION}

The seagrass, Posidonia oceanica, is present in a lot of Mediteranean sandy beach. It is crucial in protection against coastal erosion. The importance of sea grasses regarding physical aspects has been well recognized; due to their capacity to damped the wave effect. Sea grass meadows are of great importance regarding the coastal protection aspect because sea grasses have the capability to capture sediment and so to participle at the shoreline stabilization. The Sea grasses meadow slow water motion and attenuate the sediment transport in suspension. The effects of sea grasses are a lot studied on cross shore flows especially about the wave-sea grasses interaction. The degree of wave attenuation depends both on the sea grasses characteristics (the density, the height and the capacity of the shoots to bend and the wave classical parameters (wave height, period and direction). The quantification of wave energy dissipation over plants does not have a trivial expression (Mendez, 2004). Numerical modeling for such wave-sea grasses interaction is tricky because the parameters of the plant stiffness and movement under waves are difficult to define with a mathematical model. Therefore most of numerical studies approximate the grasses as to be rigid using different values for the drag coefficient (Dalrymple1984). Some experimental studies on wave dissipation due to this sea bed vegetation have been performed, with large variability of the results for wave damping over Posidonia or other meadows that confirm the difficulty to represent this flow system.

\section{DESCRIPTION OF THE FSI APPROACH WITH SPH METHOD}

The SPH method is based on the theory of interpolation (Monaghan, 1982; Monaghan, 1992; Benz, 1990; Liu, 2003; Monaghan, 2005). Its formulation is often divided into two parts: the first being the integral representation and the second "the particle approximation" (particle approximation).

The first condition is the normalization condition, the second condition is the Delta function property that is observed when the smoothing length approaches zero and the third condition is the compact condition where $K$ is a constant related to the length of smoothing and defined the effective area of the smoothing function

The stability of the SPH algorithm depends heavily on the second derivative of the kernel (Morris et al, 1997). There are different kernel functions, the most known is a Gaussian function. In this study, we use primarily the function of the third order of Monaghan and Lattanzio (Monaghan et al, 1985) known as the B-spline function.

The accuracy of the SPH method can be improved by increasing the number of neighbors, which also significantly increases the computation time. In the case of $2 \mathrm{D}$, a number of neighboring particles between 20 and 30 is a good compromise between accuracy and computation time.

\section{Fluid-solid interaction}

There are several methods to calculate fluid-structure interaction in the SPH method. Easiest method is to consider that the constraints of solid particles at the interface are directly equal to those of fluid particles. The second approach is to build an interface area between the fluid and solid particles where forces are calculated on the forces exerted by the fluid particles. These forces are then applied to

\footnotetext{
1 LEGI-UMR 5519 UJF, Grenoble University, 38041 Grenoble, France

2 LEGI-UMR 5519 UJF, Grenoble University, 38041 Grenoble, France
} 
solid particles, as well as fluid particles by interpolation at the interface. The second method is one that seems the most compatible with the code used and it is to consider the both domain (fluid and solid) as a boundary one with respect to the other to calculate the forces of action and reaction.

Solids and fluids are differentiated and those located more than $2 h$ from the interface zone are interacting only with particles of the same species (particle $a_{2}$ Fig. 1). On the other hand, the particles near the interaction zone interact with all particles. The simplest approach in this case is to extend the summations of equations for the fluid and particle part to all particles $b$ regardless of their natures.

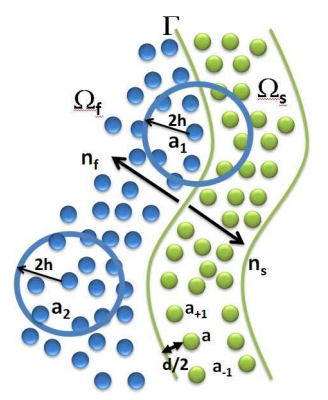

Figure 1: Fluid interaction (blue, left) solid (green, right) near the interface and convention of the normal to the interface

In principle the interpenetration of different types of particles is possible, but the correction XSPH can prevent it. This coupling method is equivalent to the conditions at the interface between the two types of particles:

$$
\begin{gathered}
u_{i f}=u_{i s} \\
\sigma_{i j s} n_{j s}=-\sigma_{i j f} n_{j f}
\end{gathered}
$$

where the subscripts $s$ and $\mathrm{f}$ represent the solids and fluids and the sign convention is that used in figure 1. Note that this approach is only valid for fluids where the viscosity is taken into account.

In the second approach (proposed by Antoci et al, 2007), the velocities of the fluids and solid particles at the interface are coupled as the constraints:

$$
\begin{gathered}
u_{i f} n_{i}=u_{i s} n_{i} \\
\sigma_{i j s} n_{i s} n_{j s}=p_{f}
\end{gathered}
$$

These equations need the precise position of the interface and the direction of the normal. One way to define the interface and the normal is given by Randles et al, (1996) However in our case, it is not necessary to take into account the fragmentation of the solid part, the latter remaining connected which makes the identification of the interface easier. The interface is defined at a distance $d / 2$ to the solid particles as shown in figure 1 (where $\mathrm{d}$ represents the distance between the initial particles) :

For all solid particles at a distance less than $2 h$ from the interface, we define the unit tangent vector:

$$
\hat{t}_{a}=\left(t_{a x} ; t_{a y}\right)=\left(\frac{x_{a+1}-x_{a-1}}{\left|\vec{x}_{a+1}-\vec{x}_{a-1}\right|}, \frac{y_{a+1}-y_{a-1}}{\left|\vec{y}_{a+1}-\vec{y}_{a-1}\right|}\right)
$$

and the unit normal vector:

$$
\hat{n}_{a}=\left(-t_{a y}, t_{a x}\right)
$$


The position of the interface for each particle is also defined by:

$$
\vec{x}_{\mathrm{int}_{a}}=\vec{x}_{a}+\left(r l+\frac{1}{2}\right) d \hat{n}_{a}
$$

where $r l$ is the number of lines between the particles $a$ and the particle interface.

\section{Definition of the condition of dynamic interface}

The condition of dynamic interface that is equivalent to the principle of action reaction is imposed by applying the fact that the force exerted by the fluid on the solid has the same magnitude as the force exerted by the solid on the fluid, but the opposite direction. For the force exerted by one phase to another, we consider the pressure gradient on the field $\Omega$ limited by the surface $\Gamma_{\Omega}$ :

$$
\langle\nabla p(\vec{x})\rangle=\left.p\left(\vec{x}^{\prime}\right) W\left(\vec{x}-\vec{x}^{\prime}, h\right)\right|_{\Gamma_{\Omega}}+\int_{\Omega} p\left(\vec{x}^{\prime}\right) \nabla_{\vec{x}} W\left(\vec{x}-\vec{x}^{\prime}, h\right) d \Omega^{\prime}
$$

which when discretized by SPH method becomes:

$$
\langle\nabla p(\vec{x})\rangle=\int_{\Gamma_{\Omega}} p\left(\vec{x}^{\prime}\right) \nabla_{\vec{x}} W\left(\left|\vec{x}-\vec{x}^{\prime}\right|, h\right) d \Gamma^{\prime}+\sum_{b} \frac{m_{b}}{\rho_{b}} P_{b} W_{a b}
$$

Assuming that the pressure varies linearly and that the support of the core is limited to a group of particle, we can define a pressure at the interface as the sum of pressure points in the length of smoothing kernel. By applying the divergence theorem and using the approximation of SPH after (Ha et al. 2011). We can rewrite the surface integral as:

$$
\begin{gathered}
F_{\text {int } a}^{f \rightarrow s}=-p_{\text {int } a} \int_{\Omega_{f}} \nabla_{\vec{x}} W\left(\vec{x}_{\text {int } a}-\vec{x}^{\prime}, h\right) d \Omega \\
F_{\text {int } a}^{f \rightarrow s}=-p_{\text {int } a} \sum_{b \in \Omega_{f}} \frac{m_{b}}{\rho_{b}} \nabla_{\vec{x}} W\left(\vec{x}_{\text {int } a}-\vec{x}_{b}, h\right)
\end{gathered}
$$

$F_{\text {int } a}^{f \rightarrow s}$ represents the intensity of the force imposed by the fluid on the point of interface and the pressure exerted by the fluid particles on the interface is interpolated by:

$$
p_{\text {int } a}=\frac{\sum_{b \in \Omega_{f}} \frac{m_{b}}{\rho_{b}} P_{b} W\left(\vec{x}_{\text {int } a}-\vec{x}_{b}, h\right)}{\sum_{b \notin \Omega_{f}} \frac{m_{b}}{\rho_{b}} W\left(\vec{x}_{\text {int } a}-\vec{x}_{b}, h\right)}
$$

$F_{\text {int } a}^{f \rightarrow s}$ can be directly brought back to the corresponding solid particle $a$ and added to the equation of motion:

$$
\frac{D v_{i_{a}}}{D t}=\sum_{b} m_{b}\left(-\left(\frac{P_{a}}{\rho_{a}^{2}}+\frac{P_{b}}{\rho_{b}^{2}}\right) \delta_{i j}+\frac{S_{i j_{a}}}{\rho_{a}^{2}}+\frac{S_{i j_{b}}}{\rho_{b}^{2}}+\Pi_{a b} \delta_{i j}+R_{i j_{a b}} f^{q}\right) \cdot \frac{\partial W_{a b}}{\partial_{a} \partial x_{j}}+g_{i}+\frac{F_{i_{a}}^{f \rightarrow s}}{\rho_{a}} \text { (13) }
$$

Finally, the reaction force on the fluid particles is interpolated using the symmetric particle of each solid particle with respect to the interface.

$$
F_{a^{*}}^{s \rightarrow f}=-F_{a}^{f \rightarrow s}
$$




$$
F_{a}^{s \rightarrow f}=\sum_{a^{*}} \frac{m_{a^{*}}}{\rho_{a^{*}}} F_{a^{*}}^{s \rightarrow f} W\left(\vec{x}_{a}-\vec{x}_{a^{*}}, h\right) \quad \vec{x}_{a} \in \Omega_{f}
$$

This reaction force is then added to the equation of motion of fluid particles:

$$
\frac{D v_{i_{a}}}{D t}=\sum_{b} m_{b}\left(-\left(\frac{P_{a}}{\rho_{a}^{2}}+\frac{P_{b}}{\rho_{b}^{2}}\right) \delta_{i j}\right) \cdot \frac{\partial W_{a b}}{\partial_{a} \partial x_{j}}+g_{i}+\frac{F_{i_{a^{*}}}^{s \rightarrow f}}{\rho_{a}}
$$

However, this method requires the creation of interface points that are difficult to create with the code. In addition, the algae did not prevent the passage of fluid particles across the frond.

The third method is based on the repulsive forces. This method draws heavily on the previous, but has the advantage of not requiring intermediate particles. It is based on the method presented by Monaghan (1994). From the normal to the solid particles calculated as previously, we consider them as boundary particles with respect to the fluid particles, that allows the calculation of reaction force. Finally, the inverse of this force is applied to solid particles. The force exerted by a fluid particle on the solid particles and calculated on the normal is given by Rogers and Dalrymple (2008):

$$
\vec{f}=n R\left(d_{\perp}\right) P(\xi) \varepsilon\left(z, u_{\perp}\right)
$$

where $n$ is the normal solid particles, the distance $d_{\perp}$ is the perpendicular distance of the particle fluid to the solid particles, $\xi$ is the projection of the distance between the solid particles and $u_{\perp}$ is the fluid velocity of the particle projected on the normal. The function of repulsion $R\left(d_{\perp}\right)$ is calculated from the normalized distance to the solid particles, $q=d_{\perp} / 2 h$, as following (modification to Monaghan and Kos (1999) original development):

$$
\begin{aligned}
R\left(d_{\perp}\right) & =A \frac{1}{\sqrt{q}}(1-q) \\
A & =\frac{1}{h} 0.01 c_{i}^{2}
\end{aligned}
$$

where $c_{i}$ is the speed of sound for the particle $i$.

The function $P(\xi)$ is chosen so that the repulsive force is constant and parallel to the particles on the edges of the solid part:

$$
P(\xi)=\frac{1}{2}\left(1+\cos \left(\frac{2 \pi \xi}{\Delta b}\right)\right)
$$

with $\Delta b$ the distance between two solid particles on edges. Finally, $\varepsilon\left(z, u_{\perp}\right)$ is used to adjust the force exerted by the fluid particle according to the height of local water $h_{0}$ and normal speed of the fluid particle:

$$
\begin{gathered}
\varepsilon\left(z, u_{\perp}\right)=\varepsilon(z)+\varepsilon\left(u_{\perp}\right) \\
\varepsilon(z)=\left\{\begin{array}{cc}
0.02 & z \geq 0 \\
\left|z / h_{0}\right|+0.02 & 0>z \geq-h_{0} \\
1 & \left|z / h_{0}\right|>1
\end{array}\right.
\end{gathered}
$$




$$
\varepsilon\left(u_{\perp}\right)=\left\{\begin{array}{cc}
0 & u_{\perp} \geq 0 \\
\left|20 u_{\perp}\right| / c_{0} & \left|20 u_{\perp}\right|<c_{0} \\
1 & \left|20 u_{\perp}\right|>c_{0}
\end{array}\right.
$$

with $z$ elevation above the water level local $h_{0}$. The normal are calculated according to equations (5 to 7). This force is then applied to the solid particles by reaction.

The code SPHysics is an open source SPH code developed by Gómez-Gesteira et al, (2010) for the study of free surfaces including waves. Several other open source codes have been developed from SPHysics, including a parallel code "parallelSPHysics' and codes using the computing power of graphics cards (GPU) as "GPUSPH". SPHysics code primarily uses the language Fortran 90 and consists of three main parts to perform all the steps required to process a case.

\section{CALIBRATION AND EXPERIMENTAL DATA COMPARISON}

The first test comparison was to calibrate and validate the Fluid Structure Interaction (FSI) that we use for the movement of algae. We compare the SPHysics results with those obtained numerically by Antoci et al, (2007) and in laboratory experiments using the SPH method. The experiment consists in the deformation of an elastic beam acting as a dam to a water column. The beam is fixed at one end and free at the other.

The experiment is described in detail by (Antoci et al, 2007). The figure below (Figure 2) shows a diagram of the experiment.

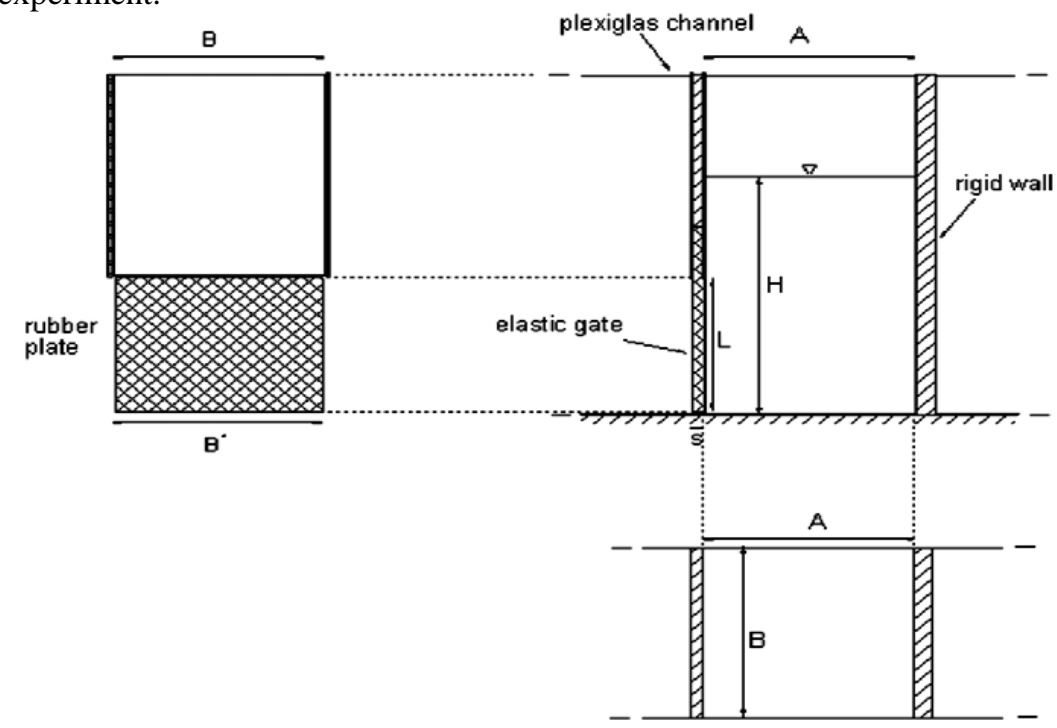

Figure 2: Schematic of the experiment conducted by Antoci et al. $200 \overline{7}$

\begin{tabular}{|l|l|}
\hline \multicolumn{2}{|c|}{ Table 1: Dimension of experiment } \\
\hline $\mathrm{A}(\mathrm{m})$ & 0.1 \\
\hline $\mathrm{H}(\mathrm{m})$ & 0.14 \\
\hline $\mathrm{B}(\mathrm{m})$ & 0.1 \\
\hline $\mathrm{B}^{*}(\mathrm{~m})$ & 0.098 \\
\hline $\mathrm{L}(\mathrm{m})$ & 0.079 \\
\hline $\mathrm{s}(\mathrm{m})$ & 0.005 \\
\hline
\end{tabular}

All dimensions of the experiment are given in Table 1. The elastic properties of the beam could not be determined precisely by Antoci et al, (2007) and the value chosen for the Young's modulus in the simulations is the one giving results closest to experiment. The data on the fluid and elastic used are summarized in Table 2: 


\begin{tabular}{|l|l|}
\hline \multicolumn{2}{|l|}{ Table 2. Properties of the fluid and the elastic moduli used } \\
\hline Fluid property & 1000 \\
\hline Density $\rho\left(\mathrm{kg} / \mathrm{m}^{3}\right)$ & $2 \times 10^{6}$ \\
\hline Compressibility modulus $\varepsilon\left(\mathrm{N} / \mathrm{m}^{2}\right)$ & \\
\hline Solid property & 1100 \\
\hline Density $\rho\left(\mathrm{kg} / \mathrm{m}^{3}\right)$ & $1.2 \times 10^{7}$ \\
\hline Young modulus $\mathrm{E}\left(\mathrm{N} / \mathrm{m}^{2}\right)$ & $4.27 \times 10^{6}$ \\
\hline Shear modulus $\mu\left(\mathrm{N} / \mathrm{m}^{2}\right)$ & $2 \times 10^{7}$ \\
\hline Bulk modulus $\mathrm{K}\left(\mathrm{N} / \mathrm{m}^{2}\right)$ & \\
\hline
\end{tabular}

To compare the results with those from the experience of Antoci, as well as with their simulations, we can trace the movement of the end of the plate (Figure 3). Note that the results match those obtained experimentally. However, as SPHysics can not use different particle sizes, we chose to use compressibility one hundred times less than that given in Table 2 to reduce the time step. Despite this difference in the compressibility, the displacements obtained seem correct (see Figure 3). Moreover, we note that the behavior of the free surface is close to the real case (Figure 4). The computation time is approximately 36 hours to 0.4 seconds of simulation

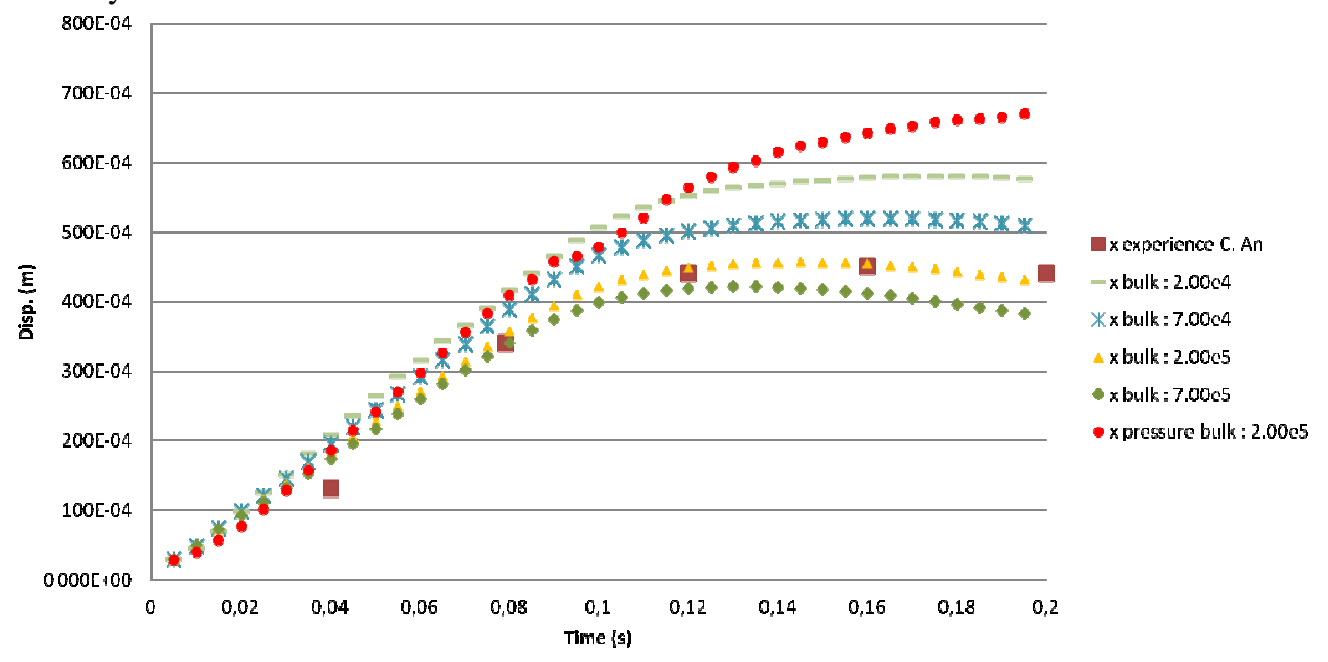

Figure 3: Horizontal displacements of the free end of the plate of the Antoci et al.(2007) experiment (ם), different value of the bulk modulus in our third SPH FSI approach (-, *, $\bullet$ and $\bullet$ ) and the test with the first approach described in this paper

To study the influence of compressibility, we have also varied its value. We can see that its influence is small, but it is difficult to know where these differences based on several elements: Antoci et al. could not determine experimentally the elastic properties of the gate. The distance between the bottom of the elastic and the ground is not given. Moreover, the method with the pressures gives permanent deformations in the plate (Figure 3, symbol $\bullet$ ) at $\mathrm{t}=0.09$ seconds, which eventually break at $\mathrm{t}=0.3$ seconds, for a displacement not more important. We therefore retain only the repulsion force method.

\section{RESULTS}

In this section we will compare the results on the movement of algae with the SPH method against experimental results. The Posidonia oceanica is an underwater plant very prevalent in the 
Mediterranean and plays an important role in mitigating the waves and sediment transport in nearshore areas by reducing the current velocity of sea floor. It has the distinction of growing in tufts, or herbarium (Figure 4), which will be a problem for the simulations

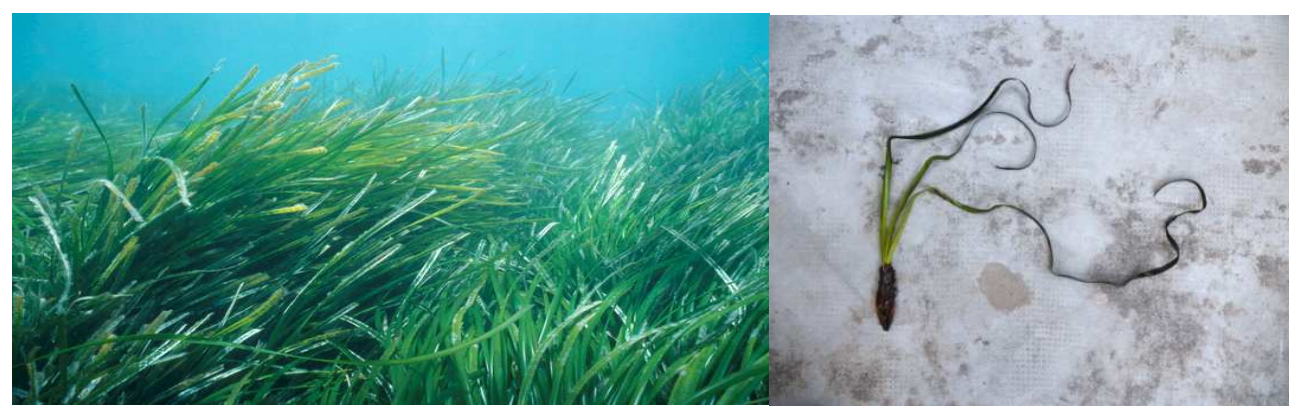

Figure 4: (left) Sea grass meadow of Posidonia oceanica and (right) photography of a plant

To model the Posidonia, it is necessary to know its properties, including its density and elastic modulus. These data have been recorded by Folkard (2005) (Table 3). However, Poisson's ratio is not known, it generally ranges from 0.3 to 0.4 for this type of solid.

To perform our simulations we have encountered several problems with the thickness of Posidonia. The first problem is that the thickness of algae is $0.1 \mathrm{~mm}$, while the particle size used in the simulations is $5 \mathrm{~mm}$. Moreover, it is necessary to model at least three rows of particles for solids in order to construct the normal to the front and therefore there is a ratio of 150 between the modeled thickness of the algae and its actual thickness. We have two options to handle this problem. We can reduce the particle size by dividing by 150 . Or modify the properties of the algae modeled. Reducing the particle size results in dramatically increasing the total number of particles and also greatly increases the computing time. In addition, since the code does not allow use of several different particle sizes, the water particles must also decrease in size. Under these conditions, the memory needed to run a calculation is too large and it is therefore impossible to use this method. We chose to use the second solution of modifying the properties of the algae. Initially, we modify only the Youngs bulk and shear's moduli and isostatic compressibility. Another study should be conducted to determine the impact on density. The new parameters are obtained directly by dividing the initial parameters by 150 (Table 3 ).

The second problem is the fact that Posidonia seagrass grow in tufts, which can not be simulated in a 2D model; however we simulate several algae close to each other, representing a broad meadow

\begin{tabular}{lll}
\hline \multicolumn{2}{l}{ Table 3. Properties of real and modified Posidonia oceanica for numerical simulations } \\
\hline Property & Real Algea & Modified Algea \\
\hline Density $\rho\left(\mathrm{kg} / \mathrm{m}^{3}\right)$ & $910 \pm 110 \mathrm{~kg} \mathrm{~m}^{-3}$ & $910 \mathrm{~kg} \mathrm{~m} \mathrm{~m}^{-3}$ \\
Young modulus E $\left(\mathrm{N} / \mathrm{m}^{2}\right)$ & $4.7 \pm 0.6 \times 10^{8} \mathrm{~N} \mathrm{~m}^{-2}$ & $3.13 \times 10^{6} \mathrm{~N} \mathrm{~m}^{-2}$ \\
Thickness $(\mathrm{mm})$ & $0.1 \mathrm{~mm}$ & $15 \mathrm{~mm}$ \\
Bulk modulus K $\left(\mathrm{N} / \mathrm{m}^{2}\right)$ & $3.92 \pm 0.6 \times 10^{8} \mathrm{~N} \mathrm{~m}^{-2}$ & $2.6 \times 10^{6} \mathrm{~N} \mathrm{~m}^{-2}$ \\
Shear modulus $\mu\left(\mathrm{N} / \mathrm{m}^{2}\right)$ & $1.81 \pm 0.5 \times 10^{8} \mathrm{~N} \mathrm{~m}^{-2}$ & $1.2 \times 10^{6} \mathrm{~N} \mathrm{~m}^{-2}$ \\
\hline
\end{tabular}

Figure 5 shows what happens when we use the first approach of FSI with the algae movement. The plants keep the deformation and can also definitely break in two or several part. The effect of the Young modulus through the change of the bulk modulus cannot avoid this breaking of the plant phenomenon. The only way to obtain a good deformation and movement is to apply the third approach for the FSI. 


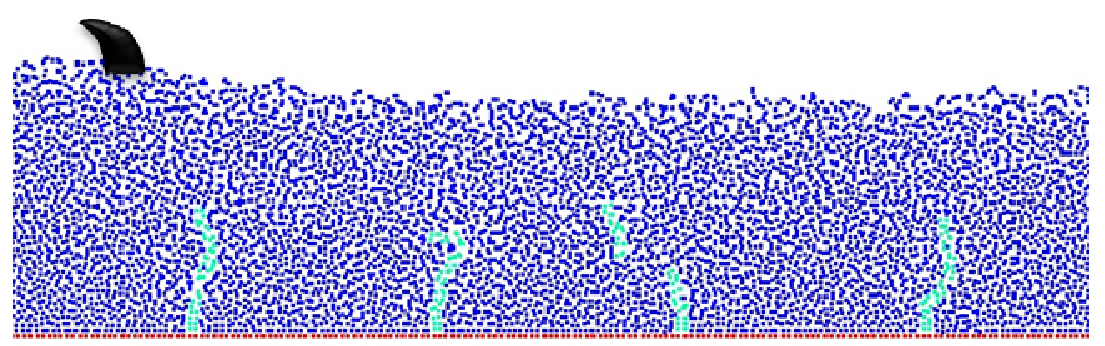

Figure 5: Movement of four plants under waves with the first approach of FSI SPH described in this paper

Luhar and Nepf (2011) studied the flow-induced reconfiguration of buoyant, flexible aquatic vegetation through a combination of laboratory flume experiments. The laboratory experiments measure drag and posture for model blades that span the natural range for seagrass stiffness and buoyancy. They also compare with a theoretical model. They test two hydrodynamic forcing: one small compares to the restoring forces and one with a forcing which exceeds the restoring forces, in this case the blades are pushed over by the flow. In this first study of ability of our approach to simulate the plant movement, we compare in a qualitative point of view our simulations with several experiments. They we constructed model blades from two different materials, silicone foam (Young modulus $\mathrm{E}=500 \mathrm{kPa} ; \Delta \rho$ $=330 \mathrm{~kg} \cdot \mathrm{m}^{-3}$ (the density of seawater is assumed to be $1025 \mathrm{~kg} \cdot \mathrm{m}^{-3}$ ) and thickness equal to $1.9 \mathrm{~mm}$ ) and high-density polyethylene, HDPE, (Young modulus $\mathrm{E}=0.93 \mathrm{GPa} ; \Delta \rho=50 \mathrm{~kg} \cdot \mathrm{m}^{-3}$ and thickness equal to $0.4 \mathrm{~mm})$.

The figure 6 shows the qualitative comparison with an experiment of Luhar and Nepf on the artificial sea plant movement under waves. Our FSI implementation in the SPH code is able to simulate the shape and the phasing of the movement due to the wave crossing over the plant. This works is the first step it has to be continuing with a more quantitative approach. The figure 7 shows that we are able also to simulate a sea grass meadow under waves.

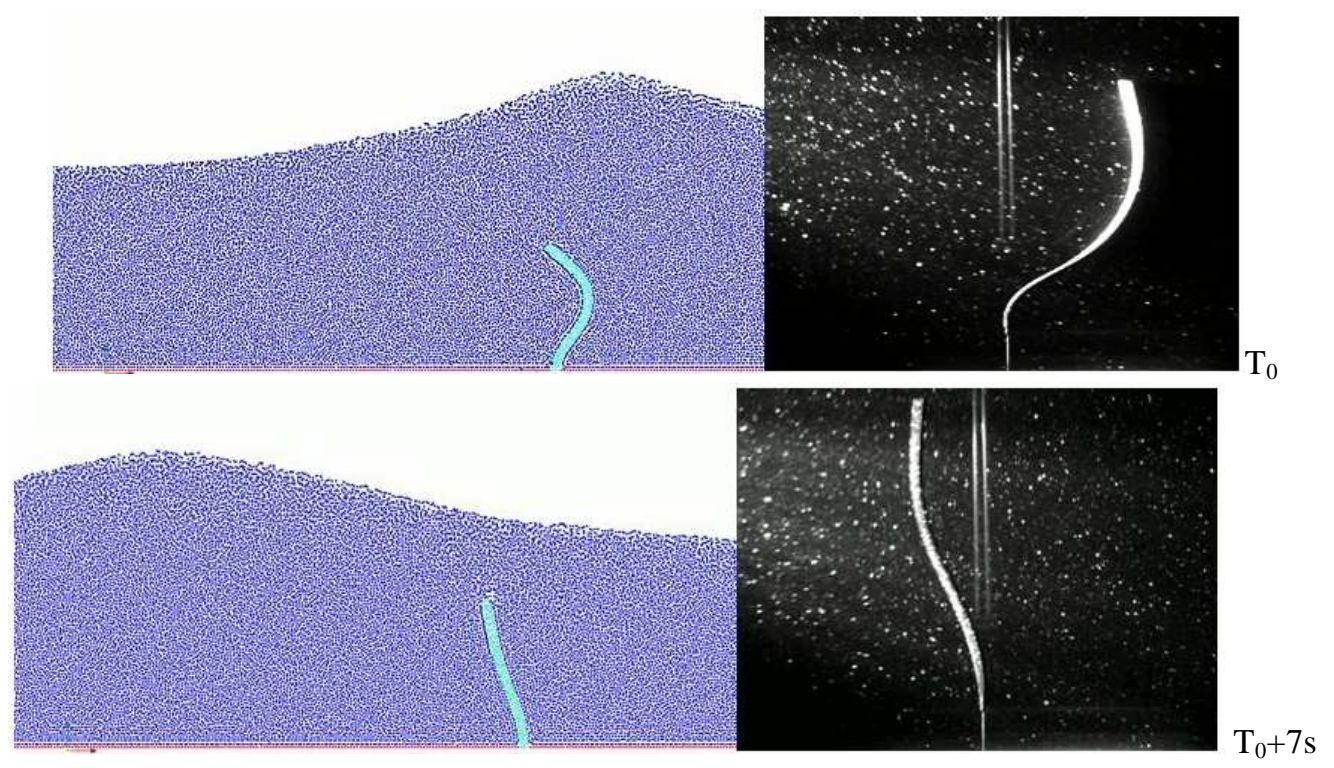

Figure 6: Movement of the artificial plant under waves at several times (left) with the third approach of FSI SPH presented in this paper and (right) Mular and Nepf experiment 


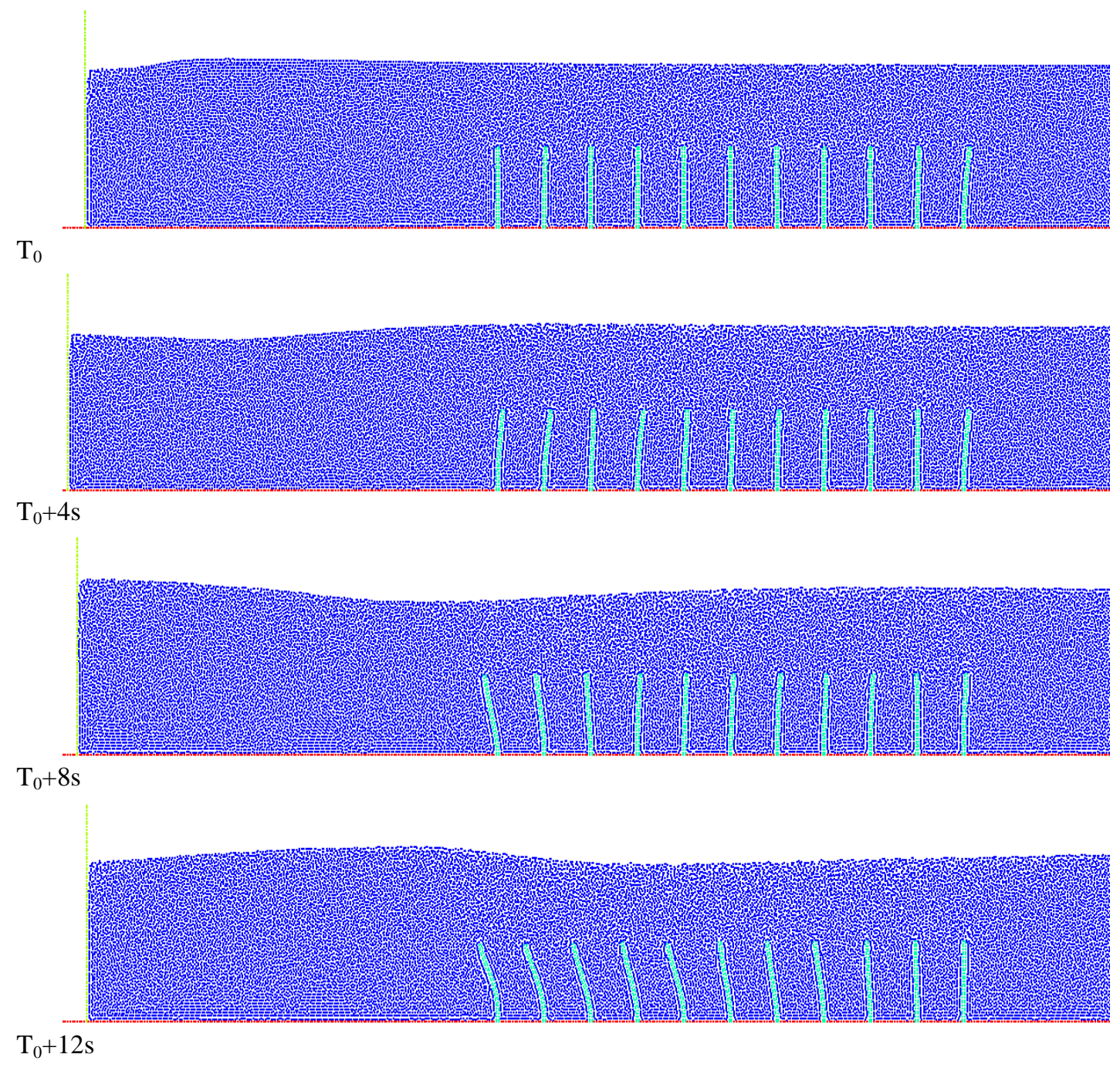

Figure 7: Movement of the sea grass meadow under waves at several times with the third approach of FSI SPH.

We compare the results for different $h_{0} / h_{\text {algae }}$ ratio $\left(h_{0}\right.$ is the reference water level in the channel) with one algae located at $\mathrm{x}=1.2 \mathrm{~m}$ (Figure 8). Figure 10 shows that the wave height generated with the piston paddle is influenced at $\mathrm{x}=0.6 \mathrm{~m}$ from the paddle and before the algae.

The height of the wave at $\mathrm{x}=1 \mathrm{~m}$ and $\mathrm{x}=1.3 \mathrm{~m}$ are nearly identical for all simulations with an increase in wave heights at the approach of algae and a significant decrease afterwards. However, when approaching the area of the channel slope (at $\mathrm{x}=1.7 \mathrm{~m}$ ), there is another increase of the wave height which varies according to the ratio $h_{0} / h_{\text {algae }}$. The presence of the algae created a decrease of the wave height, due to a lost energy by friction. This analyze requires some complementary simulations with the foot of beach slope farer from the end of the sea grass meadow to avoid the influence of it. 


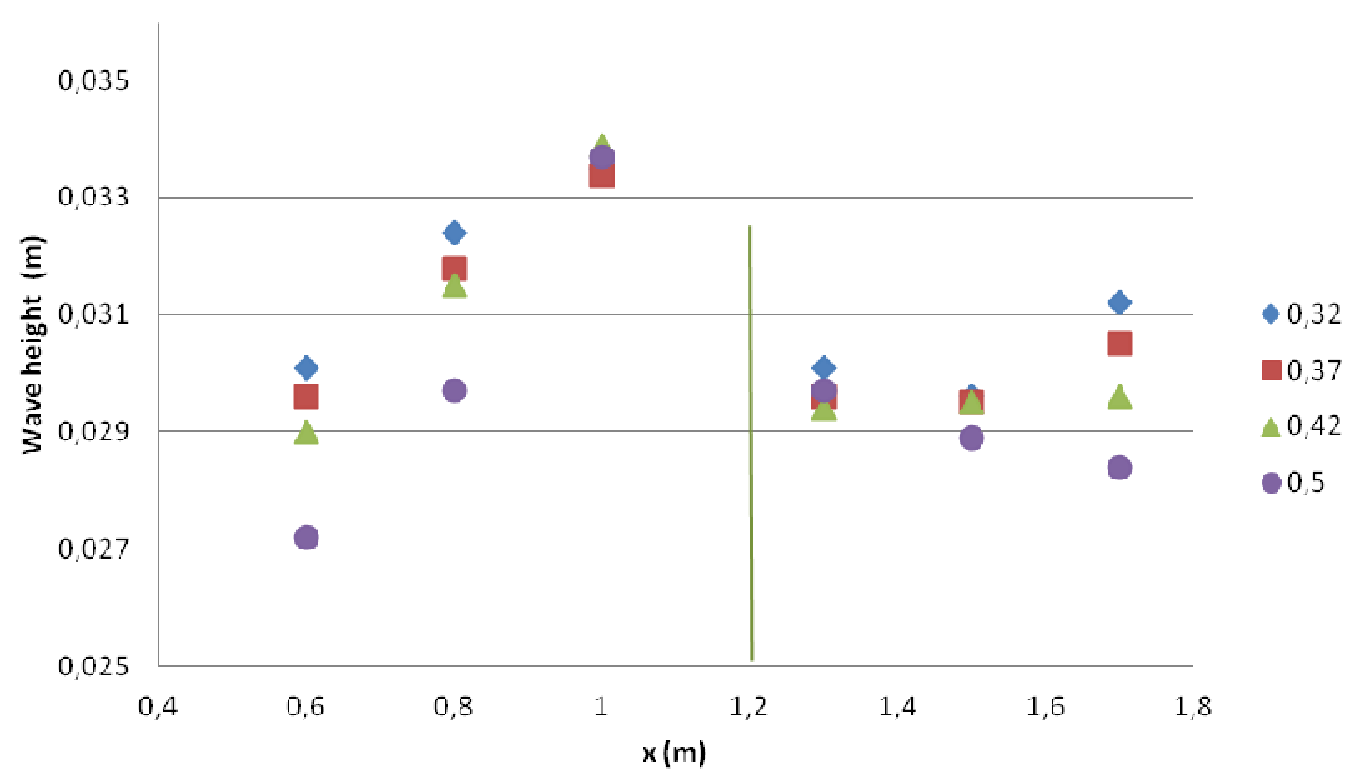

Figure 8: Wave height relative to the reference level in the channel $\left(\mathrm{h}_{0}=0.2 \mathrm{~m}\right)$ in function of the cross shore position for the ratio $\mathrm{h}_{0} / \mathrm{h}_{\text {algae }}$ ranging from 0.32 to 0.5 . The green line shows the algae's position at $\mathrm{x}=1.2$ meters.

\section{CONCLUSIONS}

The SPH with FSI gives reasonable results for the various simulations, although several improvements are still needed. Indeed, we showed using a test case of deformation of a elastic plate over the a water column, the SPH method, with fluid solid interaction model worked and gave the results for the movement of the solid part. Next we simulate the movement of algae in a numerical wave flume. The different curves on the attenuation of swells, we can say that the presence of algae does play an important role. In our first simulation over a meadow the damping of the wave height is significant but we need more numerical experiment with longer and/or higher sea grass meadow to compare with existing experiment in the literature. The influence of non-rigid behavior of the algae was highlighted by the comparison of results. However the results obtained for the attenuation of waves remain qualitatively given the sizing problems mentioned in the previous chapter. Moreover, the cost in computation time of the SPH method is too important to perform simulations on very large areas now, but should determine an equivalent viscosity that would be used in simulations using other method. If the attenuation of waves obtained numerically for Posidonia is identical to the in-situ measurements that will simulate the influence of any type of algae, and determine an equivalent viscosity based on its properties.

Although the initial results seem consistent with expectations, many improvements are possible especially in terms of comparison with experimental results, but also in terms of computation time in order to launch 3D calculations. The results of numerical simulations are currently difficult to compare with experimental results given the differences of initial parameters due to the limitations of the SPH method, particularly related to computation time. One of the first improvements might be to compare more than simple theoretical cases, dimensions numerically feasible, with the displacement of the solid and the fluid part of the known, to determine whether to add a coefficient in the law Interaction. The second element is the computation time is very important for the SPH method. One of the futur improvements would be to use the code GPUSPH which has the advantage of being an open source SPH code which also uses the immense computational power of graphics card.

\section{ACKNOWLEDGMENTS}

This work has been supported by ANR KUNSHEN. We highly appreciate the discussions M.P. Cani from INRIA and S. Meulé from the CEREGE. We also thank M. Luhar and H. Nepf (from the MIT) for the exchange of information and data. 


\section{REFERENCES}

Agamloh E.,Wallace A., von Jouanne A., “Application of Fluid Structure Interaction Simulation of an Ocean Wave Energy Extraction Device," American Institute of Aeronautics and Astronautics 25th Wind Energy Symposium (including revolutionary ocean energy concepts), January 2006

Amini Y., Emdad H. and Farid M.(2011) A new model to solve fluid-hypo-elastic solid interaction using the smoothed particle hydrodynamics (SPH) method, European Journal of Mechanics B/Fluids 30 pp.184-194

Antoci C., Gallati M. and Sibilla S., (2007), Numerical simulation of fluid-structure interaction by SPH, Computers and Structures 85 pp. 879-890

Crespo, A.J., M. Gómez-Gesteira, and R.A. Dalrymple, "Boundary Conditions Generated by Dynamic Particles in SPH Methods", CMC: Computers, Materials, \& Continua, 5, 3, 173-184, 2007.

Dalrymple R.A., Kirby J.T. and Hwang P.A. (1984). 'Wave Diffraction Due to Areas of Energy Dissipation', Journal of Waterway, Port, Coastal, and Ocean Engineering, Vol. 110, Issue 1, pp. 6779.

Dalrymple, R.A. and B.D. Rogers, "Numerical Modeling of Water Waves with the SPH Method," Coastal Engineering, 53/2-3, 141-147, 2006.

Farhat, C., Van der Zee K.,Geuzaine P., Provable second-order time-accurate loosely-coupled solution algorithms for transient nonlinear computational aeroelasticity, J. Comput. Methods Appl. Mech. Engrg., 195, pp. 1973-2001 (2006)

Fonseca M.S., Cahalan J.H. (1992). 'A preliminary evaluation of wave attenuation by four species of Seagrass', Estuarine, Coastal and Shelf Science, 35 (6), pp. 565-576.

Folkard, A.M. 2005 Hydrodynamics of model Posidonia oceanica patches in shallow water, Limnology and Oceanography. 50: 1592-1600

Gingold, R.A. Monaghan, J.J. 1977 Smoothed particle hydrodynamics: theory and application to nonspherical stars, Mon. Not. R. Astron. Soc. 181: 375-389.

Gómez-Gesteira, M. Rogers, B.D. Dalrymple, R.A., Crespo A.J.C. and Narayanaswamy, M. 2010, User Guide for the SPHysics Code v2.0, http://wiki.manchester.ac.uk/sphysics

Gray, J.P. Monagahn, J.J. Swift, R.P. 2001. SPH elastic dynamics. Comp Methods Appl Mech Eng; 190: 6641-6662.

Ha, Y.D. Kim, M.G. Kim, H.S and Cho, S. 2011 Shape design optimization of SPH fluid-structure interactions considering geometrically exact interfaces, Struct Multidisc Optim, 44:319-336.

Hérault, A. Bilotta, G. Dalrymple, R.A. Rustico, E. Del Negro, C. GPUSPH (Version 2.0) (Software) Available from www.ce.jhu.edu/dalrymple/GPUSPH

Kobayashi N., Raichlen A.W., Asano T. (1993). 'Wave Attenuation by Vegetation', Journal of Waterway, Port, Coastal, and Ocean Engineering, Vol. 119, Issue 1, pp. 30-48.

Le Tallec, P. Mouro, J. 2000 Fluid structure interaction with large structural displacements. Comput Methods Appl Mech Eng 2000:1-29.

Leimkuhler, B. J. Reich, S. Skeel, R.D. 1997 Integration Methods for Molecular dynamic IMA Volume in Mathematics and its application. Springer

Liu, G.R. 2003. Mesh Free methods: Moving beyond the finite element method. CRC Press, 692.

Lucy, L.B. 1977. A numerical approach to the testing of the fission hypothesis, Astron. J. 82: 10131024.

Luhar, M. and Nepf, H. M. 2011 Flow-induced reconfiguration of buoyant and flexible aquatic vegetation, J of Limnology and Oceanography, 56: Issue 1, 2003-2017.

Mendez, F.J. and Losada I.J. 2004 An empirical model to estimate the propagation of random breaking and nonbreaking waves over vegetation fields, Coastal Engineering, 51: Issue 2, 103-118.

Monaghan, J. J. 1982. Why particle methods work. Siam J. Sci. Stat. Comput. 3: 422-433.

Monaghan, J. J. Gingold, R.A. 1983. Shock simulation by the particle method SPH. J. Comp. Phys. 52: 374-389.

Monaghan, J.J. Lattanzio, J.C., 1985. A refined method for astrophysical problems. Astron. Astrophys. 149: 135-143.

Monaghan, J. J. 1989. On the problem of penetration in particle methods. J.Computational Physics, 82: 1-15.

Monaghan, J. J. 1992. Smoothed particle hydrodynamics. Annual Rev. Astron. Appl., 30: 543- 574.

Monaghan, J. J. 1994. Simulating free surface flows with SPH. Journal Computational Physics, 110 : 399- 406. 
Monaghan J. J. and Kocharyan A. 1995. SPH simulation of multi-phase flow. Computer Physics Communication, 87:225-235.

Monaghan, J. J. and Kos, A. 1999. Solitary waves on a Cretan beach. J. Wtrwy. Port, Coastal and Ocean Engrg., 125: 145-154.

Monaghan, J.J., Cas, R.A.F. Kos, A.M. Hallworth, M. 1999 Gravity currents descending a ramp in a stratified tank. J Fluid Mech, 379:39-69.

Monaghan, J. J. 2005. Smoothed Particle Hydrodynamics. Rep. Prog. Phys. 68: 1703-1759.

Morris, J. P. Fox, P. J. Zhu, Y. 1997. Modeling low Reynolds number incompressible flows using SPH, J. Comp. Phys. 136: 214-226.

Prinos, P. Koftis, T. Galiatsatou, P. 2010 Wavelet analysis of wave propagation over Posidonia oceanica. Proceedings on the Third International Conference on the Application of Physical Modelling to Port and Coastal Protection, COASTLAB

Randles, P.W. Libersky, L.D. 1996 Smoothed particle hydrodynamics: some recent improvements and applications. Comput Methods Appl Mech Eng ; 139 :375-

Rogers, B.D. and Dalrymple, R.A. 2008 SPH Modeling of tsunami waves, Advances in Coastal and Ocean Engineering, Advanced Numerical Models for tsunami waves and run-up, World Scientific. 10

Rugonyi, S. Bathe, K.J. 2001 On finite element analysis of fluid flows fully coupled with structural interactions. Comput Model Eng Sci; 2: 195-212.

Sánchez-González, J.F. Sánchez-Rojas, V. Memos, C.D. 2011 Wave attenuation due to Posidonia oceanica meadows, Journal of Hydraulic Research Vol. 49, Iss. 4

Stratigaki, V., Manca, E., Prinos, P. (2010). Effects of Posidonia oceanica meadow on wave propagation, Large-scale experiments. Proc. 4th Int. Short Conf. Applied coastal res. Barcelona, 230-240.

Stratigaki V., Manca E., Prinos P., Losada I.J., Lara J.L., Sclavo M., Amos C.L., Cáceres I. and Sánchez-Arcilla A.(2011): Large-scale experiments on wave propagation over Posidonia oceanica, Journal of Hydraulic Research, 49:sup1, 31-43

Ward L.G., Kemp W.M., and Boynton W.E. (1984). 'The influence of waves and seagrass communities on suspended particulates in an estuarine embayment', Marine Geology, Vol. 59, pp. 85-103. 